\title{
Analysis of Collaborative Learning in a Computational Thinking Class
}

\author{
Bushra Chowdhury \\ Department of Engineering Education \\ Virginia Tech \\ bushrac@vt.edu
}

\author{
Austin Cory Bart \\ Department of Computer Science \\ Virginia Tech \\ acbart@vt.edu
}

\author{
Dennis Kafura \\ Department of Computer Science \\ Virginia Tech \\ kafura@vt.edu
}

\begin{abstract}
Collaborative learning can help reduce the anxiety level of learners, improve understanding and thus create a positive atmosphere for learning. This study analyzes students' collaborative learning experiences within small interdisciplinary "cohorts" while learning computational thinking in a universitylevel class. The cohort allows students from different disciplines to contribute diverse perspectives, socially interact with each other and in turn create situations where two or more students learn together. This study uses both qualitative and quantitative means to explore students' collaborative learning experiences. Ethnographically-informed qualitative data using Stahl's collaborative framework is analyzed. The analysis revealed that most students found the cohort model to be valuable in learning computational thinking by allowing them to ask about and explain problems, especially with students from different disciplines who perceive and explain a problem differently. Quantitative data from a multi-term survey complements and confirms the findings from the qualitative data. Our study helps to inform those teaching foundational computing concepts to a diverse audience of learners.
\end{abstract}

\section{CCS CONCEPTS}

- Human-centered computing $\rightarrow$ Collaborative and social computing $\rightarrow$ Collaborative and social computing design and evaluation methods $\rightarrow$ Ethnographic studies.

\section{KEYWORDS}

Computational thinking, collaborative learning, cohort, interdisciplinary groups

\section{ACM Reference format:}

Bushra Chowdhury, Austin Cory Bart, Dennis Kafura, 2018. Analysis of Collaborative Learning in a Computational Thinking Class. In Proceedings of the ACM SIGCSE conference, Baltimore, Maryland, USA. February 2018 (SIGCSE'18), 6 pages.

\section{INTRODUCTION}

Computational Thinking (CT) has caught the attention of a broad academic community $[1$, p.38]. Wing defined "computational thinking" as "a universally applicable attitude

Permission to make digital or hard copies of all or part of this work for personal or classroom use is granted without fee provided that copies are not made or distributed for profit or commercial advantage and that copies bear this notice and the full citation on the first page. Copyrights for components of this work owned by others than ACM must be honored. Abstracting with credit is permitted. To copy otherwise, or republish, to post on servers or to redistribute to lists, requires prior specific permission and/or a fee. Request permissions from Permissions@acm.org.

SIGCSE'18, February 21-24, 2018, Baltimore, MD, USA.

(C) 2018 Association of Computing Machinery.

ACM ISBN 978-1-4503-5103-4/18/02..\$15.00.

DOI: http://doi.org/10/1145/3159450.3159470 and skill set everyone, not just computer scientists, would be eager to learn and use" [2, p.33]. Being able to think computational has been identified as a major skill " $21^{\text {st }}$ Century" [3] in part because nearly three out of four jobs will require significant CS skills and knowledge [5. As the availability of big data and integration of computing in different disciplines increases, the necessity of people in diverse disciplines with the ability to think computationally has also increased. Thus different initiatives for integrating CT within existing curricula at various academic levels have started to gain footing.

Educators in the field of computer science are grappling with the challenge of teaching their discipline to an audience more diverse in their backgrounds and goals than has been traditional. This is even more the case for teaching CT to undergraduates who are unlikely to pursue a career in computer science and may not have a strong analytical background. Thus, it is important to assess the pedagogical approach of teaching CT to non-CS majors.

Collaborative learning has emerged as a technique that researchers have found to be generally applicable and effective for teaching Computer Science concepts [6,7]. Collaboration can help reduce the anxiety level of learners, improve understanding and thus create a positive atmosphere to learn CT [8]. In collaborative learning particular forms of interaction are expected to occur which would trigger learning [9, p.5]. During collaboratively learning, individuals negotiate and share meanings relevant to the problem-solving task at hand [10, p. 70]. Collaborative interactions between students majoring in different disciplines can thus promote not only a disciplinary notion of CT but also an interdisciplinary notion of CT that fosters transfer of computational concepts across disciplines [11].

This study, focusing on experiences of non-computer science majors enrolled in a general education CT course, makes two contributions:

- $\quad$ presents a multi-disciplinary collaborative learning "cohort model" appropriate for CT, and

- $\quad$ shows the value of this model by analyzing the experience of students using ethnographic and survey methods.

This paper gives lengthier treatment to the ethnographic study in order to convey more vividly the cohort learning experience.

The cohort model has five features:

Multi-disciplinary: Each cohort had students from multiple majors. The cohorts were assigned by the instructor. A strong attempt was also made to achieve gender balance with no cohort having fewer than two female students. 
Persistent: Students were assigned to a cohort in the second week of the semester and the cohort lasted throughout the remaining 14 weeks of the semester. The longevity of the cohort allows students to develop a degree of social acceptance that lowers the barriers to collaboration.

Active peer learning: Each class day students sit together as a cohort at a table and perform all class room activities within their cohort. Students were strongly encouraged and guided in how to interact with their cohort members. A cohort "contract" was developed and signed by each cohort at the beginning of the semester. This contract outlined their responsibilities to the cohort and to each other. Students could also collaborate virtually using the course book, a custom-built, interactive webbased platform with embedded coding activities and real-time, shared text writing.

Individual evaluation: The class room activities, as well as virtually all other course work, was done individually but freely provided and received clarification, explanation, and encouragement to others in their cohort.

No cohort evaluation: Believing that a cohort assessment would interfere with its social dynamics, no assessment or selfassessment of the cohort members' level or quality of participation was done. This allows students to participate at whatever level they are comfortable with. It is possible for a student to engage minimally or not at all and still receive as high a grade as someone who more fully interacted in their cohort.

The collaborative learning that occurred within the cohort model is analyzed as follows. The qualitative methods (Section 2) use Stahl's major influences of collaborative learning as a lens to study the student's experiences [12]. The results are presented (Section 3) and discussed (Section 4). The quantitative survey and its results are given in Section 5. The survey analysis complements the ethnographic findings but is independent, having been conducted over a different and larger student population in a different semester. Conclusions are given in Section 6.

\section{Theoretical Framework and Research Design}

Studies focusing on collaborative learning usually consider group of learners collaboratively solving a task together. In this study, the "cohort" approach of collaboratively learning was slightly different because students in a cohort were solving the same problem individually at the same time. The cohort was designed to act as a supportive sub community within the classroom setting, taking more of naturalistic peer learning model. Thus, the study required a research framework that was flexible enough to incorporate such variations of collaborative learning. According to Stahl, the framework is not intended to be a model of objects and processes but rather illustrates how members of small groups can engage in a cognitive activity. Hence the framework seemed suitable for this particular study which allowed the researcher to focus on students' experiences of learning in general as well as the collaborative features emerging from their experiences.

\subsection{Stahl's major influences of Collaborative Learning}

Various factors of a setting and the participant's personal attributes influence collaborative learning. Group members'

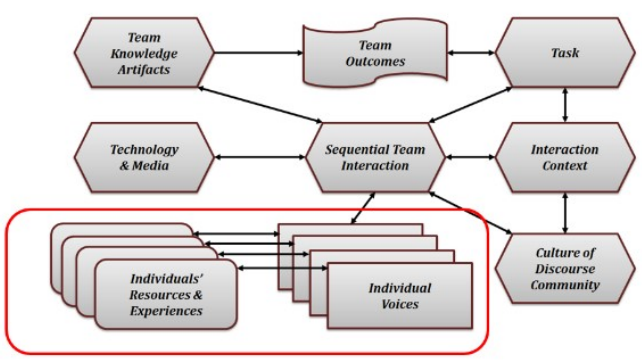

Figure 1 Major influences of collaborative learning [12]

prior knowledge, perspective, abilities, understanding of the task to be completed, the technology and media used, the context of interactions and, finally, the culture of the community, all play vital roles in the collaborative process. Error! Reference source not found. illustrates the major influences of collaborative learning as described by Stahl [12].

According to Stahl's framework, all the major influences in a collaborative learning environment interact with the sequential team interaction goal. In the context of learning CT in a classroom setting, interactions between learners, learners and the instructors, and learners and the course materials can be considered as collaborative interactions. These interactions weave all the other influences to each other. Among other influences, a student's background, prior knowledge and perspective specifically impact how $\mathrm{s} / \mathrm{he}$ interacts in a collaborative environment. This study focuses on these major influences on student learning CT collaboratively, particularly on "individual' resources and experiences". According to Stahl, "much of the power of collaborative learning can come from the pooling of different knowledge and alternative perspectives distributed within the group" (page 257).

Thus, in order to better understand the role disciplinary background and how it shapes a student's experience in learning CT collaboratively, ten undergraduate students (majoring in different non-computer science) who had enrolled in a CT course were interviewed.

\subsection{Research Design}

The goal of this study was to investigate how students in a CT course experience collaboration. The study used ethnographically-informed data collection methods (observations, interviews and surveys). Interviews as a data collection method allows a researcher to gain better understanding of people's experiences and perceptions. Researchers focusing on social interactions have interviewed participants to know how the participants felt doing an activity, and to reflect on their experiences [14]. This study thus mainly focuses on qualitative data coming from semi-structured interviews in order to gain better understanding on student's perceptions of peer interactions in the general education CT class. The study also collected quantitative survey data. 
The study was conducted in a state university in the Southeast U.S. As part of the strategic plan the university has been offering a general education course titled "Introduction to CT" to students from all disciplines. In spring 2015, when the ethnographic study was conducted the class enrolled approximately 45 students and met twice a week for 75 minutes sessions. Each class began with a 10-15 minute review of the days topic followed by active class work. A short (5-10 minutes) discussion period concluded the class.

In order to better understand major influences experienced by students while learning collaboratively, members of three of the eight cohorts (Error! Reference source not found.) were individually interviewed in the last two weeks of the semester. A total of 10 students were interviewed. The semi structured interviews typically lasted 15 to 40 minutes were audio recorded and transcribed. The interview protocols were informed by Stahl's collaborative learning framework, primarily focusing on students' background, goals, interests, and experiences while learning CT. The interview participants were voluntary. The researcher obtained Human Subjects Research Approval through the Institutional Review Board (IRB) of the corresponding University before initiating the interview for the study. Consent of the participants was obtained prior to the initiation of data collection. The full interview protocol can be accessed at https://think.cs.vt.edu/resources/ct-protocol.

Table 1: Profile of students interviewed

\begin{tabular}{|c|c|c|c|}
\hline Cohort & $\begin{array}{l}\text { No. of students } \\
\text { interviewed }\end{array}$ & $\begin{array}{l}\text { Total no. of } \\
\text { students in the } \\
\text { cohort }\end{array}$ & Major \\
\hline $\begin{array}{l}\text { Cohort } \\
\text { Alpha }\end{array}$ & $\begin{array}{l}3 \text { (senior:2 } \\
\text { freshman:1) }\end{array}$ & 5 & \multirow{3}{*}{$\begin{array}{l}\text { History, } \\
\text { Political } \\
\text { Science, } \\
\text { Architecture, } \\
\text { Chemistry, } \\
\text { Public } \\
\text { Relations, } \\
\text { Housing, } \\
\text { Theater } \\
\text { Arts, } \\
\text { Journalism }\end{array}$} \\
\hline $\begin{array}{l}\text { Cohort } \\
\text { Beta }\end{array}$ & $\begin{array}{l}\text { 4(senior:3, } \\
\text { sophomore:1) }\end{array}$ & 6 & \\
\hline $\begin{array}{l}\text { Cohort } \\
\text { Gamma }\end{array}$ & $\begin{array}{l}\text { 3(sophomore:2, } \\
\text { freshman:1) }\end{array}$ & 6 & \\
\hline
\end{tabular}

All transcribed audio recording of individual student interviews were coded using a prior code informed by Stahl's collaborative learning framework. Two independent researchers coded separately for the purpose of cross-checking coding and emerging themes. Discrepancies with codes were discussed and resolved. To protect the anonymity, pseudonyms were assigned to each of the interview participant.

\section{RESULTS}

A brief summary of the findings are given and then explained in detail. First, for most of the non-science majors, learning to code was learning something conceptually different than they were used to. These students struggled to write code, finding it difficult to comprehend how precise one had to be. Nevertheless, most of the students could comprehend the value of learning CT and its applicability to their own discipline. Second, most of them found the long term cohort model to be valuable resources in learning CT. Asking and explaining problems to cohort members were not only useful to advance through a problem but also valuable to one's own learning process. Discussing problems with students coming from different disciplines allowed them to see how differently students perceive and explain a problem. Students coming with a science background could recognize the struggle non-science majors faced while learning to code. Third, providing ample time for students to actively learn CT during class time, acknowledging that students will make mistakes, allowing students to casually communicate with each other, and the overall structure of the course were few salient features of the CT course that students brought up during their interviews.

The following sections summarize student's personal CT learning experiences according to the major influences highlighted in Stahl's framework.

3.1 Individual's Resources and Experiences. All students of this study were taking the CT course to fulfill a general education requirement in quantitative reasoning. A course in calculus was the most common class taken by student to satisfy this Area 5 requirement prior to the offering of this CT course. Most of the participants found math difficult and the option of taking something different encouraged them to take the CT class.

Students majoring in different disciplines were able to relate the use of CT to their fields. For some students, the final project of the CT class helped them to make this connection. For example, a student in journalism was able to describe how big data is being used to analyze and predict crime-related behaviors. A student in Public Relations understood that $\mathrm{s} / \mathrm{he}$ might need to apply his/her computation skill while developing websites. The student majoring in chemistry explained how computational analytics help chemists to discover new drugs. The following excerpts are taken from the conversation between the journalism, architecture and chemistry majors respectively: "It's sort of on the cusp of journalism is now, they're going through very large things of data. Most recently, the Chicago red light thing that was going on with red light cameras in Chicago. Somebody had to go through all 4 million instances of red light tickets. And you could go through them manually, or you can have a programmer go through all of it. If you can do that as a journalist it's getting more and more to where you kind of need to have a niche position. Almost as if the job has never been there before, like Washington Post or something like that. Somebody's going through all that data to create the graphs that end up on the news." [Journalism Major]

"I'll probably be able to use it ... The coding, the different rules for coding. I'm actually working on a website in another of my classes so it's helpful to know how coding works. Then also just how we're working on the visualizations for the final project right now, I think that's going to be pretty helpful in the future to be able to analyze data in my PR I'll probably be able to use that." [Architecture Major]

However, some students still had a very rudimentary perception on CT and were not able elaborate on the applicability of CT in their own field. For example, in the following quote, a History major gives a very brief definition of CT: 
"I would just say using computers to analyze data." [History Major]

The profiles of the interviewed participants revealed that students who were more advanced in the study of their discipline were able to elaborate on the connection of $\mathrm{CT}$ to their own discipline while freshman and sophomore were not clear in making the connection. This difference could be explained by the fact that the freshman and sophomore students were new to their own disciplines and their disciplinary perspectives were not fully formed.

3.2 Individual voices (Role of a student). Students can take on various roles in a collaborative environment [13]. In this study the roles students assumed were oriented towards peer learning in a cohort where each student was solving the same problems individually but could voluntarily ask help from their cohort members.

The main roles students conveyed in their interviews were asking a question, responding to a question and taking the role of a mentor. Most of the students interviewed explained their role in the cohort as both giving and receiving help. Students would work on the problems by themselves and if they got stuck, they would ask help from a cohort member.

"During the class we would work on our own and if we had questions we would ask each other sometimes I would ask, sometimes someone else would ask me" [Architecture Major]

One particular student, Joe (not his real name), assumed more of a mentor role. Joe had prior programming experience and was willing to help others. As a result he was seen helping out most of the other students in his cohort. His cohort members also found his presence to be assuring. In the following quote, a Liberal Arts major describes Joe's role:

"I really liked our group. We would just work on our stuff and mostly ask questions, we'd go to foe because he's the only one who has any CS background. So he knows everything compared to us. We would ask him a lot of questions. He's a super helpful guy and super talkative...I had lots of learning opportunities, from foe especially and then from other people much less so. I think also another reason that everybody was here all the time is because we had foe. He had that kind of background experience. Even if nobody else was getting it, Jonathan gets it. I think we always felt safe to come to class" [Liberal Arts Major]

Joe describes his first couple of weeks of CT mentoring experience as follows:

"I wasn't really prepared to take on this role, so I wasn't thinking I need to be a leader. It was sort of like oh, I know about this stuff and you guys don't really know all that much about this stuff, I guess I should help you out, and it was sort of a last minute thing. It kind of shook me for the first couple of weeks. I was like these people really need my help." [Science Major]

3.3 Culture of discourse community. The students believed the cohort model to be beneficial to learning. The students appreciated the in-class time allowed to learn CT with cohort members.

"I think it's because we were provided ample time every single class to work as a group... I think that was probably the biggest facilitator of the group interaction was just being in the same place at the same time at a time that was convenient for everyone, because we were planning on being there for class." [English Major]

The Journalism Major appreciated the cohort as a natural conversational mode with cohort members.

"I guess I was really fond of the way we could just casually bring up the conversation instead of formally raising your hand, and the class would stop ... I just felt like it was more natural conversation." [Journalism Major]

One of the main reasons why students found the cohort model to be helpful is to have someone at the same level of learning explain a problem.

"Mostly because we're all kind of on similar levels of understanding, my cohort members and I, so when they explain it in their own words, it's just easier for me to understand" [History Major]

According to the History major it is helpful when a learner with the same level of understanding explains a problem in their own words.

"And for me, I personally would rather hear a student's point of view on a problem because I probably relate to them more than an instructor. Usually the student will give some sort of example to coincide with the problem. So, I guess it's not as hard to grasp. While the instructor will use more of the technical terms which I probably don't know so, it's harder for me to grasp the concept.'[Political Science Major]

A Political Science major preferred an explanation from a peer student rather than the instructor. This particular student found the use of technical terms by the instructor to be difficult to interpret. In the excerpt bellow a student majoring in Theater Arts describes that at times when he was burdened thinking over a problem another cohort member would explain the problem in more basic terms which was helpful.

"Like if I was more confused, if I was overthinking a problem in something, my cohort members would boiled it down to more bare basics. That was helpful." [Theater Arts Major]

In the two excerpts below a student majoring in English and another majoring Architecture describe that explaining a problem to another cohort member can be helpful for one's own understanding.

"A lot of times we would just say the things we were doing, especially in [the block-base language], as we were doing them. I've definitely learned, not just in this class but overall as a psychology thing, being able to explain back something that you've been told is a really good way of gauging understanding. It's always funny when you ask a question to our group because you'll get some really interesting answers. Part of them will be funny and part of them will be legitimate, but we all shared the struggle I guess." [English Major]

"Others helping me helps me learn it through, like on a one-to-one scale with a person my age. It's kind of relatable. So you're learning with someone who's learning with you, and if they know something, it's like oh, I can learn it too. And if I know something they don't, they can learn it too, and we're just helping each other grow. Of course, if I help him with something, and I can verbally communicate it with him that means that I'm understanding it more, and that's definitely a learning skill." [Architecture Major] 
The Architecture major in the above excerpt elaborates on the aspect of enhancing self-confidence. Seeing someone with similar experience in learning CT able to solve a problem helped him to believe that he could also solve the problem.

Liberal Arts students found learning CT to be a different type of learning then they were used to. For example:

"I don't get this. I'm not used to having to be so precise and so logical about everything." [Political Science Major]

When the student was asked to elaborate on what he meant by "being precise and logical" the Political Science Major elaborated, "There is more than one way to write the same thing. Even if your sentence is not fully formed others can figure out what you want to say. But with computers, it has to be picture perfect, or else it doesn't work."

"I'm not really good with ... My brain's more of the English and history side versus science and math" [English Major]

Students appreciated the exchange of ideas with cohort members and having students coming from different disciplines in their cohort.

"Students coming from different discipline had a different take on how they explained a problem or solution of a problem and that was nice". [Political Science Major]

3.4 Interaction context. The students mentioned that it was important not only to talk about and solve problems in a cohort but to get to know each other, attend class regularly, tell a joke and laugh about things happening in each other's lives. These non-academic conversations made them feel comfortable to ask questions to each other and probably also kept the cohort functional.

"Building that sense of community, just friendship and working relationships with the people in my cohort was such a huge part I think for us of how we got along so well, just little things here and there. We weren't just coming to do the group work, we were all getting to know each other and I think that really helped." [English Major]

Students appreciate the long lasting cohort model for the community aspect it provided for the students:

"Since we're able to all talk about it, like we're comfortable sharing our problems or our complications with each other, it was easy to learn like that, because it was just like you weren't afraid to ask for help, and sometimes, your cohort members would explain something a different way, and then it would help you understand it." [History Major]

3.5 Technology and media. Students in the CT class did not only communicate with their cohort members within class but also on Facebook. They had a Facebook group where they communicated, shared their experiences, problems they were having with home works and help one another outside the class room.

"We have a Facebook message for if we're doing homework or something and we need help that we're all linked into so we all see it. We can then message, "Oh, well I see your code. You really need to put a parenthesis here. You're not counting this right," or something like that. We can all help each other, not while we're even just in the classroom. We can meet outside the classroom." [Science Major]

\section{DISCUSSION}

In studying small group cognition, Stahl suggested focusing on the role, interactions and discourse between group members [15]. In this study, students' experiences while learning CT were also influenced by other factors that contribute to small group cognition. Students' role and the discourse within the CT class were shaped by the resources that were available, and the interactions student had with their peers, the instructor and the TAs. Fig. 3 maps Stahl's collaborative framework to factors influencing the experiences of students.

The findings reveal that most students taking this CT class

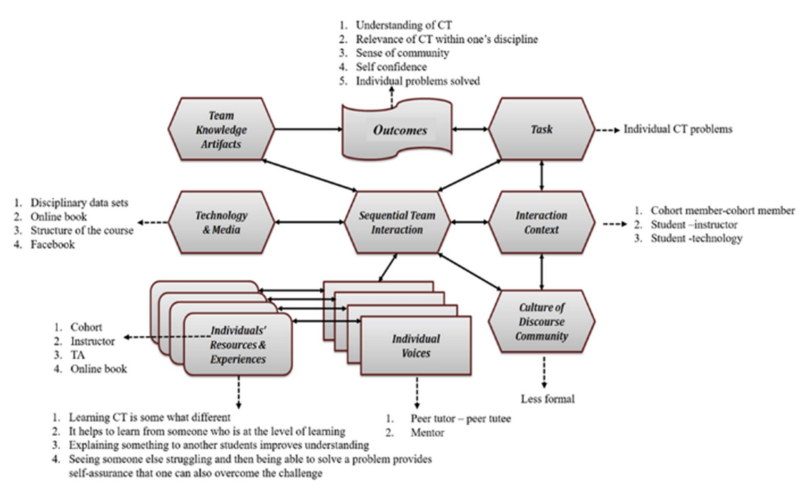

Figure 3: Factors influencing non-computer science majors' experiences in learning CT within small groups

did not have prior experience in learning computational concepts and were enrolled in non-STEM majors. Only a few students had some prior programming experience. Most of the students were taking this course as alternative to a math course (calculus). Thus, student's discipline and prior experience in computing were considered as the background of the participants. The discourse in the small groups as well in the class was less formal. Students could casually start talking to one another.

In terms of available resources, students found having a semester long small groups (cohort model) helpful to learning CT. Students characterized their role in the small groups as a tutee (mostly asking questions) or as a tutor (providing explanations). Some students also characterized their role as mentors. Students found it beneficial to explain and be able to see how others solved a problem. The students found it comforting that they were able to work on a data set from their own discipline. The disciplinary data sets allowed the students to bring in their own disciplinary knowledge and combine what they had learned from the CT class.

In terms of experiences, students described learning CT to be different than typical type of learning they were commonly used to. Breaking down problems to its very basic level so that a computer can understand seemed difficult for them to comprehend. For some students learning to code was like learning another language. However, for a student majoring in science, learning CT was not that much different.

In terms of outcome, students referred to being able to comprehend what $\mathrm{CT}$ is, the applicability of $\mathrm{CT}$ within one's 
own discipline, the ability to write a computer program and having a sense of community within the class.

4.1 Implications for practice. Students with a non-science background can find learning CT difficult. On the other hand, the process of learning $\mathrm{CT}$ is more intuitive and comes naturally to the students coming from a science background with prior programing experience. Using discipline specific datasets can be helpful to keep students interested and motivated to learn CT. Forming semester long groups/cohorts can be beneficial for novice learners. These small groups provide students a sense of belonging, a place to share thoughts and anxieties. Students find it is easier to naturally ask questions to group member than help from instructors. An explanation coming from a peer who is at the same learning level is easier to understand and relate to. Educators should also keep in mind that some students may struggle and need extensive support.

4.2 Limitations and future directions Students who participated in this study were self-selected. For this reason, it is likely that most of them had a positive attitude towards the CT class. Also, most of the students interviewed in this study were taking this particular CT course to avoid taking a course in 'math'. This could have influenced the findings of this study. There were disciplines from which students were enrolled in the course but were not interviewed. Interviewing students from a larger number disciplines could have allowed the researcher to gain a broader perspective. A future direction of this work could be to investigate the similarities and dissimilarities within student's disciplinary backgrounds that support or hinder learning of CT for undergraduate students.

\section{QUANTITATIVE ANALYSIS}

Complementing the ethnographic analysis a survey was conducted over two semesters (Fall 2016 and Spring 2017). The survey was voluntary, had no weight in the course grade, and was done under an IRB approved protocol. A total of 176 out of 191 students (a $91 \%$ response rate) gave consent to participate in the study. Approximately $60 \%$ of the students were female. There was a distribution over academic years (freshmen and sophomores were each $30 \%$ of the class while juniors and seniors were each 20\%).

The survey asked six questions which are identified in Figure 4 as: Giving (I felt comfortable giving help to others in my cohort.), Interaction (I felt the others in my cohort often interacted with me and the others.), Learning (I felt my cohort helped my learning.), Seeking (I felt comfortable getting help from others in my cohort.), Useful (I believe that the course material is useful.), and External/Useful (I felt the others in my cohort believed that the course material is useful). Each question was answered on a 7 point Likert scale as shown in the figure.

The survey shows an overall strong and positive response to the cohort experience. Students felt extremely comfortable in giving assistance and only slightly less comfortable in seeking assistance. A small minority was less than comfortable seeking assistance though none were uncomfortable giving assistance. The frequency of interaction was rated slightly higher than the contribution to learning though the positive view of each was extremely strong. It is interesting to note that individuals believed more strongly that the material was useful then they perceived this to be the case of others in their cohort.

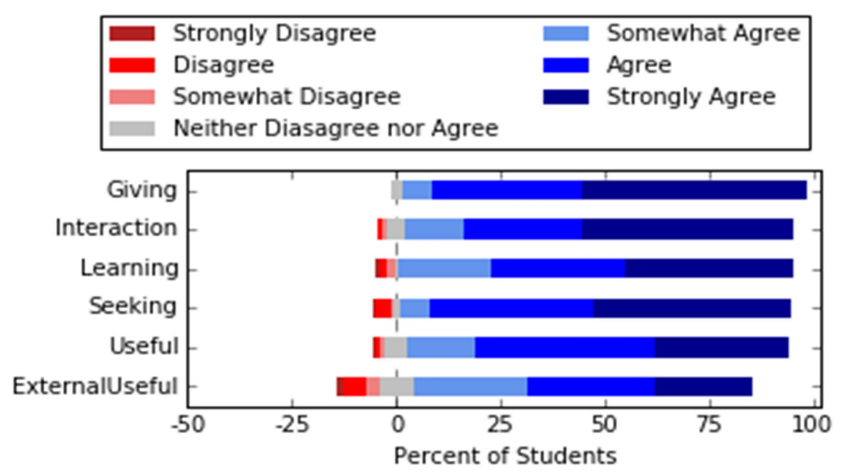

Figure 4 Results of Survey Data

\section{CONCLUSIONS}

Findings of this study suggest that novice CT learners from different disciplines had varied experiences in learning CT. The students valued the cohort model of learning CT collaboratively. Disciplinary data sets allowed students to combine their disciplinary knowledge with computational skills learned from the CT class. The students also valued having a learning environment where they could learn from peers who they have come to know and trust. Taken together, these results suggest the benefits of collaborative learning and the need for further mixed-methods analyses of how students work together to learn about computational thinking

\section{REFERENCES}

[1] Grover, S. and R. Pea, Computational Thinking in K-12 A Review of the State of the Field. Educational Researcher, 2013. 42(1): p. 38-43.

[2] Wing, J., Computational thinking. Communications of the ACM, 2006. 49(3): p. 33-35.

[3] National Research Council, A framework for K-12 science education: Practices, crosscutting concepts, and core ideas. 2012: National Academies Press.

[4] Education for Life and Work: Developing Transferable Knowledge and Skills in the 21st Century, ed. W.P. James and L.H. Margaret. 2012: The National Academies Press.

[5] Bureau of Labor Statistics, U.S. Department of Labor. Occupational Outlook Handbook, 2016-17. Computer and Information Research Scientists; Available from: https://www.bls.gov/ooh/computer-and-informationtechnology/computer-and-information-research-scientists.htm (visited June 03, 2017).

[6] McDowell, C., et al. The effects of pair-programming on performance in an introductory programming course. in ACM SIGCSE Bulletin. 2002. ACM.

[7] Kavitha, R. and M.I. Ahmed, Knowledge sharing through pair programming in learning environments: An empirical study. Education and Information Technologies, 2015. 20(2): p. 319-333.

[8] Hmelo-Silver, C.E., The international handbook of collaborative learning. 2013: Routledge.

[9] Dillenbourg, P., What do you mean by collaborative learning? Collaborativelearning: Cognitive and Computational Approaches., 1999: p. 1-19.

[10] Roschelle, J. and S.D. Teasley. The construction of shared knowledge in collaborative problem solving. in Computer supported collaborative learning. 1995. Springer.

[11] Lattuca, L.R., L.J. Voigt, and K.Q. Fath, Does interdisciplinarity promote learning? Theoretical support and researchable questions. The Review of Higher Education, 2004. 28(1): p. 23-48.

[12] Stahl, G., Guiding group cognition in CSCL. International Journal of Computer-Supported Collaborative Learning, 2010. 5(3): p. 255-258.

[13] Wasserman, S. and K. Faust, Social network analysis: Methods and applications. Vol. 8. 1999: Cambridge university press.

[14] Belbin, M., Belbin team roles. Book Belbin Team Roles, 2004.

[15] Stahl, G., Theories of Cognition in Collaborative Learning, in International Handbook of Collaborative Learning, C. Chinn, et al., Editors. 2013. p. 74-92. 